\title{
In-Situ Study of Nb Hydride for SRF Cavity Applications Using Aberration-Corrected STEM and Electron Energy Loss Spectroscopy
}

\author{
R. Tao ${ }^{1}$, A. Romanenko ${ }^{2}$, and L.D. Cooley ${ }^{2}$, R. F. Klie ${ }^{1}$ \\ 1. Department of Physics, University of Illinois at Chicago, Chicago, IL, 60607 USA \\ 2. Fermilab, P.O. Box 500, Batavia, IL 60510 USA
}

Niobium is a type-II superconducting material with the highest critical temperature $\left(\mathrm{T}_{\mathrm{c}}=9.2 \mathrm{~K}\right)$ and generally used to fabricate superconducting radio-frequency (SRF) cavities for linear particle accelerators. We present an atomic-resolution study of the effects that a 48 hour bake at $120^{\circ} \mathrm{C}$ in vacuum has on the high-field properties of Nb-based SRF cavities. This bake results in a significant increase in high-field quality factor Q. However, an $800{ }^{\circ} \mathrm{C}$ bake for 2 hour reduces the $\mathrm{Hc}_{3} / \mathrm{Hc}_{2}$-ratio of cavities. Several mechanisms have been proposed to explain this contrary behavior, including an increased $\mathrm{NbO}_{\mathrm{x}}$ surface layer thickness and the precipitation of $\mathrm{NbHy}$.[1]

Using a combination of atomic-resolution Z-contrast imaging and electron energy-loss spectroscopy with in-situ heating and cooling experiments, we examine the atomic and electronic structures of $\mathrm{Nb}$ and related oxides/hydrides near the cavity surface. Also, we demonstrate that hydrogen atoms, incorporated into the $\mathrm{Nb}$ crystal by forming $\beta-\mathrm{NbH}$ precipitates, can be directly visualized using annular bright field imaging in our aberration-corrected JEOL ARM-200CF as shown in Figure 1(a). Image calculations of the $\beta-\mathrm{NbH}$ phase are performed using the STEM image simulation code by Kirkland, which employs the fast-Fourier-transform multislice algorithm. Figure 1(b) shows the experimental and calculated intensity profile for $\beta-\mathrm{NbH}$ [110]. [2]

Atomic resolution imaging can also be achieved during in-situ cooling. Figure 1(c) shows the ABF image of $\mathrm{Nb}$ [100] at $\mathrm{LN}_{2}$ temperature. We can see a weak image contrast at the center of the square formed by the $\mathrm{Nb}$ atomic columns. Next, we use EEL spectroscopy to quantify the hydride phases, in particular the $\beta, \varepsilon$ and $\zeta$-phases, which have different crystal structures and hydrogen concentrations. In Figure 2 , the spectrum at room temperature shows the typical metallic $\mathrm{Nb}$ features with the broad peak at $10.5 \mathrm{eV}$, however, at $\mathrm{LN}_{2}$ temperature the peak at $10.5 \mathrm{eV}$ disappears and is replaced by a peak at $7.8 \mathrm{eV}$.

In an effort to further improve the performance of $\mathrm{Nb}$ SRF cavities, a wide range of treatments are attempted during the manufacture process, which result in different defect structures at the $\mathrm{Nb}$ surface, as well as variety of impurities. Such defects and impurities will further reduce the superconductivity of the $\mathrm{Nb}$ cavities. Figure 3(a) shows $\mathrm{ABF}$ and $\mathrm{HAADF}$ images of $\mathrm{Nb}$ from the cavity surface after growing thin $\mathrm{NbN}$ layer on the inside of the cavity. We find that the $5 \mathrm{~nm}$ thick oxide layer is still present, and, from high-resolution $\mathrm{ABF}$ image shown in Figure 3 (b), the nitrogen atoms are visible inside of $\mathrm{Nb}$ crystal. Image simulation will also be presented.

In this presentation we will examine the effects of impurities and defects on the atomic and electronic structures of $\mathrm{Nb}$ as a function of the different treatment methods. Our results are helpful for predicting SRF cavity performance and to evaluate future manufacturing processes.[3]

\section{Reference}

1. R. Tao, R. F. Klie et al, Journal of Applied Physics, 110, Issue 12, (2011) 
2. Y.J. Kim, R. Tao, R.F. Klie and D. Seidman, ACS nano 7 (1), pp 732-739, (2013)

3. This work is supported by the University Research Associate (URA) Visiting Scholars Program at the Fermi National Accelerator Laboratory. The acquisition of the UIC JEOL JEM-ARM200CF is supported by an MRI-R ${ }^{2}$ grant from the National Science Foundation (Grant No. DMR-0959470).
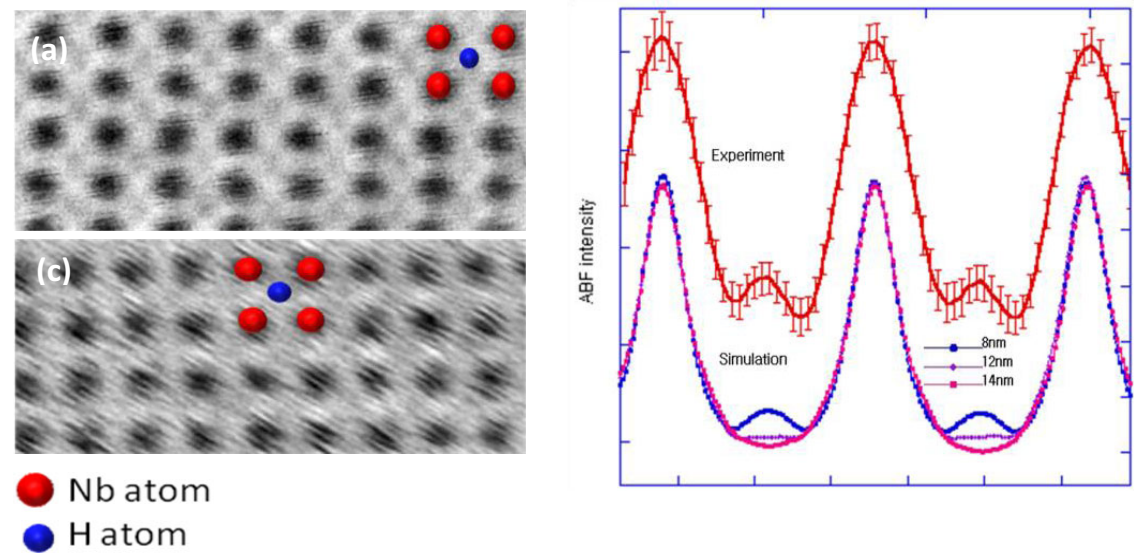

Figure 1: (a) ABF image of [10] direction $\beta-\mathrm{NbH}$ phase with visible Hydrogen atoms, (b) intensity profile plot comparison between experimental and simulation results, (c) ABF image of $\mathrm{Nb}$ [100] direction at $\mathrm{LN}_{2}$ temperature

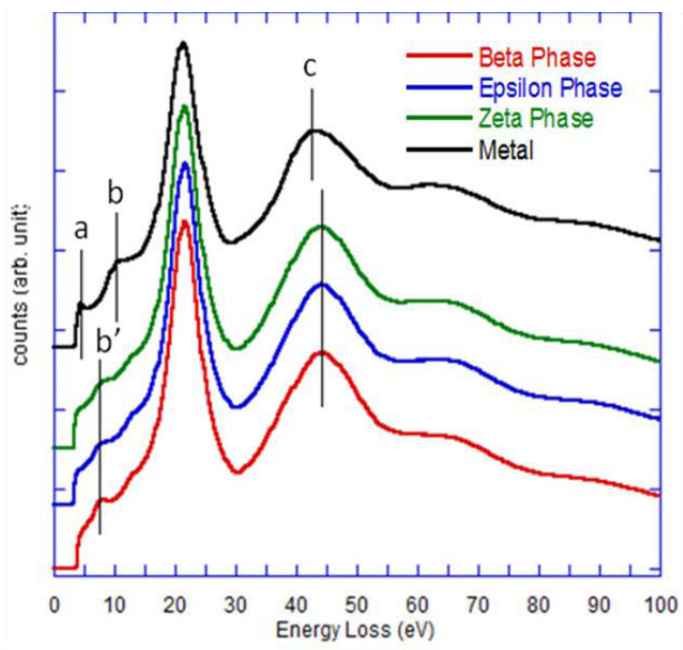

Figure 2: summary of $\mathrm{Nb}$ metal and three $\mathrm{NbH}$ phases low-loss peak after extracting zero-loss peak
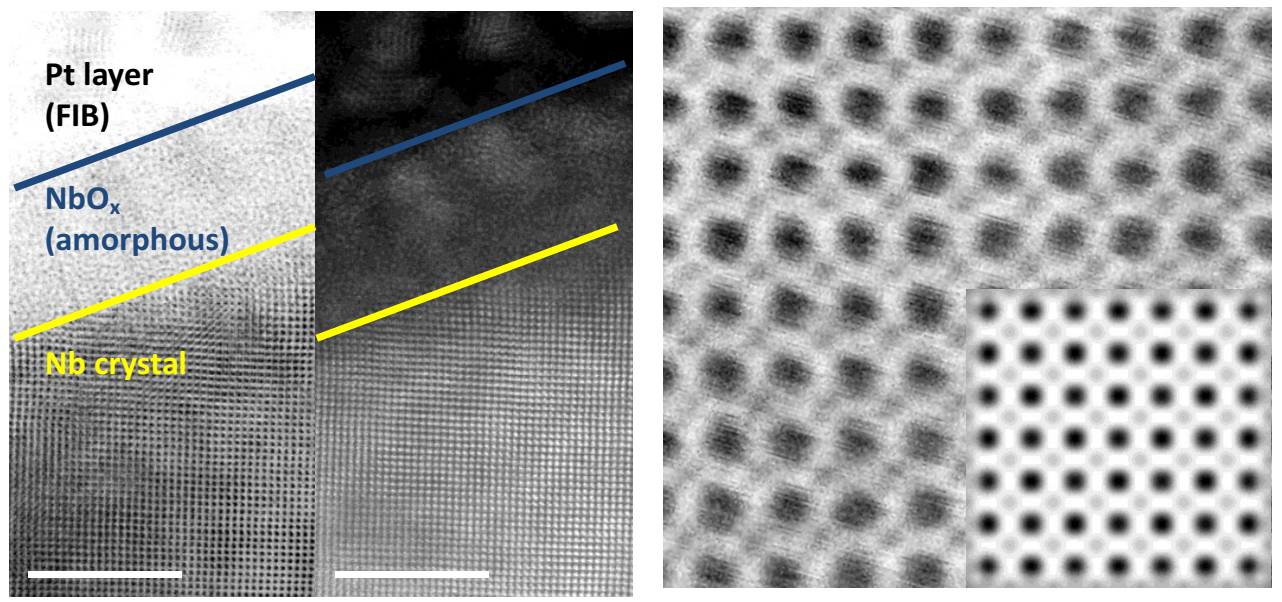

Figure 3: (a) ABF and HAADF image of $\mathrm{Nb}$ cavity surface after attempting at growing thin $\mathrm{NbN}$ layer, (b) ABF image shows visible nitrogen atoms in $\mathrm{Nb}$ crystal of $\mathrm{NbN}$ and corresponding Kirkland code simulation of image 